SV

\title{
ON THE IN-PLANE VIBRATION AND STABILITY OF A SPINNING ANNULAR DISK
}

\author{
Jen-SAN Chen AND JHI-Lu Jhu \\ Department of Mechanical Engineering, National Taiwan University, Taipei, Taiwan 107, \\ Republic of China
}

(Received 10 July 1995, and in final form 22 January 1996)

\begin{abstract}
A comprehensive investigation of the free in-plane vibration of a spinning annular disk is presented in this paper. Emphasis is given to the effect of clamping ratio on the natural frequencies and critical speeds of the spinning disk. For a disk with small clamping ratio, the limiting speed at which the disk is allowed to rotate safely is determined by the critical speed of the axisymmetrical mode. Beyond this critical speed divergence instability will be induced. For a disk with large clamping ratio, on the other hand, the speed limit is determined by the critical speed of a mode with a large number of nodal diameters. It is observed that the critical speeds of the modes with nodal diameters approach an asymptotic value as the number of nodal diameters increases. Numerical simulations show that this asymptotic critical speed is independent of the clamping ratio, while it is dependent on the Poisson ratio of the disk.
\end{abstract}

(C) 1996 Academic Press Limited

\section{INTRODUCTION}

It is well known that the natural frequencies of the in-plane radial and torsional vibrations of a spinning disk depend on the rotational speed. Bhuta and Jones [1] investigated the axisymmetric planar vibration of a rotating disk and found that effect of rotation is generally to lower the natural frequencies. The same problem was studied by Doby [2] with a different formulation. Burdess et al. [3] generalized the analysis to asymmetric in-plane vibration, and discussed the properties of both the forward and backward travelling circumferential waves. In most of these works the disk is assumed to be full, and as a consequence only two boundary conditions on the outer radius should be satisfied. A comprehensive analysis of the stability and natural frequencies of in-plane vibration of a spinning annular disk, which should be of more practical importance, cannot be found in the literature.

In this paper we first derive the equations of motion for the spinning disk with respect to a stationary co-ordinate system. Lamé's potentials are used to simplify the highly coupled equations. Natural frequencies for annular disks with various clamping ratios are presented. Emphasis is given to the effects of the clamping ratio on the natural frequencies and critical speeds of the spinning disk.

\section{EQUATIONS OF MOTION}

In Figure 1 is shown an elastic annular disk spinning at constant speed $\Omega$. The material of the disk is assumed to be homogeneous and isotropic, with mass density $\rho$, Young's modulus $E$ and Poisson ratio $v$. If the disk is thin, plane stress conditions can be assumed and the equilibrium equations in the radial and tangential directions in terms of the 
in-plane displacements $u_{r}$ and $u_{\theta}$ can be written with respect to the body-fixed co-ordinate system $(r, \theta)$ as [3]

$$
\begin{gathered}
\left\{E / \rho\left(1-v^{2}\right)\right\}\left(L_{11} u_{r}+L_{12} u_{\theta}\right)=\ddot{u}_{r}-2 \Omega \dot{u}_{\theta}-\Omega^{2}\left(u_{r}+r\right), \\
\left\{E / \rho\left(1-v^{2}\right)\right\}\left(L_{21} u_{r}+L_{22} u_{\theta}\right)=\ddot{u}_{\theta}+2 \Omega \dot{u}_{r}-\Omega^{2} u_{\theta},
\end{gathered}
$$

where the differential operators are defined as

$$
\begin{array}{cc}
L_{11}=\frac{\partial^{2}}{\partial r^{2}}+\frac{1}{r} \frac{\partial}{\partial r}-\frac{1}{r^{2}}+\frac{1-v}{2 r^{2}} \frac{\partial^{2}}{\partial \theta^{2}}, & L_{12}=\frac{1+v}{2} \frac{1}{r} \frac{\partial^{2}}{\partial r \partial \theta}-\frac{3-v}{2} \frac{1}{r^{2}} \frac{\partial}{\partial \theta}, \\
L_{21}=\frac{1+v}{2} \frac{1}{r} \frac{\partial^{2}}{\partial r \partial \theta}+\frac{3-v}{2} \frac{1}{r^{2}} \frac{\partial}{\partial \theta}, & L_{22}=\frac{1-v}{2}\left(\frac{\partial^{2}}{\partial r^{2}}+\frac{1}{r} \frac{\partial}{\partial r}-\frac{1}{r^{2}}\right)+\frac{1}{r^{2}} \frac{\partial^{2}}{\partial \theta^{2}} .
\end{array}
$$

Equations (1) and (2) can be rewritten with respect to an inertial frame $(r, \eta)$ by using the transformations

$$
\dot{u}_{r}(r, \theta)=\frac{\partial u_{r}(r, \eta)}{\partial t}+\Omega \frac{\partial u_{r}(r, \eta)}{\partial \eta}, \quad \dot{u}_{\theta}(r, \theta)=\frac{\partial u_{\eta}(r, \eta)}{\partial t}+\Omega \frac{\partial u_{\eta}(r, \eta)}{\partial \eta},
$$

where

$$
\eta=\theta+\Omega t
$$

It is convenient at this stage to introduce some dimensionless variables and operators (denoted with an asterisk):

$$
\begin{gathered}
r^{*}=r / b, \quad \zeta=a / b, \quad u_{r}^{*}=u_{r} / b, \quad u_{\eta}^{*}=u_{\eta} / b, \\
\Omega^{*}=\Omega b \sqrt{\rho / E}, \quad t^{*}=(t / b) \sqrt{E / \rho} .
\end{gathered}
$$

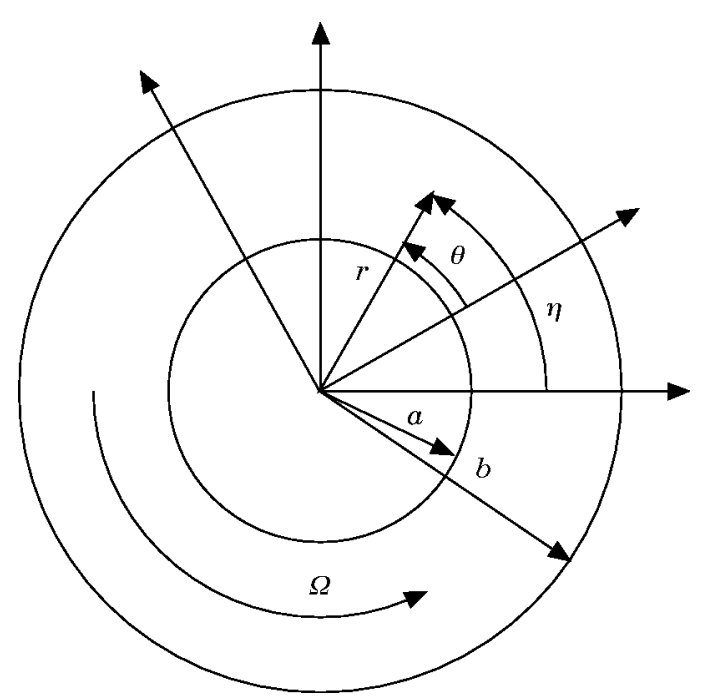

Figure 1. A freely spinning annular disk. 
The equations of motion in terms of these dimensionless variables with respect to the inertial frame can be written as follows:

$$
\begin{aligned}
\frac{1}{\left(1-v^{2}\right)}\left(L_{11}^{*} u_{r}^{*}+L_{12}^{*} u_{\eta}^{*}\right)= & \left(\frac{\partial^{2} u_{r}^{*}}{\partial t^{* 2}}+2 \Omega^{*} \frac{\partial^{2} u_{r}^{*}}{\partial t^{*} \partial \eta}+\Omega^{* 2} \frac{\partial^{2} u_{r}^{*}}{\partial \eta^{2}}\right) \\
& -2 \Omega^{*}\left(\frac{\partial u_{\eta}^{*}}{\partial t^{*}}+\Omega^{*} \frac{\partial u_{\eta}^{*}}{\partial \eta}\right)-\Omega^{* 2}\left(u_{r}^{*}+r^{*}\right), \\
\frac{1}{\left(1-v^{2}\right)}\left(L_{21}^{*} u_{r}^{*}+L_{22}^{*} u_{\eta}^{*}\right)= & \left(\frac{\partial^{2} u_{\eta}^{*}}{\partial t^{* 2}}+2 \Omega^{*} \frac{\partial^{2} u_{\eta}^{*}}{\partial t^{*} \partial \eta}+\Omega^{* 2} \frac{\partial^{2} u_{\eta}^{*}}{\partial \eta^{2}}\right) \\
& +2 \Omega^{*}\left(\frac{\partial u_{r}^{*}}{\partial t^{*}}+\Omega^{*} \frac{\partial u_{r}^{*}}{\partial \eta}\right)-\Omega^{* 2} u_{\eta}^{*} .
\end{aligned}
$$

The differential operators $L_{11}^{*}, L_{12}^{*}, L_{21}^{*}$ and $L_{22}^{*}$ are similar to those in equations (3)-(6) with $r$ and $\theta$ being replaced by $r^{*}$ and $\eta$, respectively. We assume that the annular disk is clamped at the inner radius $r^{*}=\zeta$ and is free at the outer boundary $r^{*}=1$. The dimensionless boundary conditions with respect to the inertial frame are

$$
\begin{gathered}
u_{r}^{*}=u_{\eta}^{*}=0 \quad \text { at } r^{*}=\zeta, \\
\frac{1}{1-v^{2}}\left[\frac{\partial u_{r}^{*}}{\partial r^{*}}+v\left(\frac{u_{r}^{*}}{r^{*}}+\frac{1}{r^{*}} \frac{\partial u_{\eta}^{*}}{\partial \eta}\right)\right]=0, \frac{1}{2(1+v)}\left(\frac{1}{r^{*}} \frac{\partial u_{r}^{*}}{\partial \eta}+\frac{\partial u_{\eta}^{*}}{\partial r^{*}}-\frac{u_{\eta}^{*}}{r^{*}}\right)=0 \quad \text { at } r^{*}=1 .
\end{gathered}
$$

It is noted that the differential equation (10) is inhomogeneous due to the body force term $r^{*} \Omega^{* 2}$. As the disk rotates at certain speed, steady in-plane displacements $u_{r}^{*(s)}\left(r^{*}, \eta\right)$ and $u_{\eta}^{*(s)}\left(r^{*}, \eta\right)$ are induced due to the centrifugal effect. We consider small perturbations in displacements, $\hat{u}_{r}^{*}\left(r^{*}, \eta ; t^{*}\right)$ and $\hat{u}_{\eta}^{*}\left(r^{*}, \eta ; t^{*}\right)$, superposed on the steady state solutions. By substituting the total displacements, $u_{r}^{*(s)}+\hat{u}_{r}^{*}$ and $u_{\eta}^{*(s)}+\hat{u}_{\eta}^{*}$, into the equilibrium equations (10) and (11), one can obtain the equations of vibration in terms of the small displacement perturbations $\hat{u}_{r}^{*}$ and $\hat{u}_{\eta}^{*}$. The resulting equations and boundary conditions are similar to equations (10)-(13) with $r^{*} \Omega^{* 2}$ in equation (10) being deleted and displacements $u_{r}^{*}$ and $u_{\eta}^{*}$ being replaced by $\hat{u}_{r}^{*}$ and $\hat{u}_{\eta}^{*}$, respectively. In the following discussion we neglect all the asterisks and drop the superposed hats on $\hat{u}_{r}^{*}$ and $\hat{u}_{\eta}^{*}$ for brevity.

\section{LAMÉ'S POTENTIALS}

The homogeneous equations (10) and (11) can be simplified if Lamé's potentials $\phi$ and $\psi$ are introduced [4]:

$$
u_{r}=\frac{\partial \phi}{\partial r}+\frac{1}{r} \frac{\partial \psi}{\partial \eta}, \quad u_{\eta}=\frac{1}{r} \frac{\partial \phi}{\partial \eta}-\frac{\partial \psi}{\partial r}
$$

Substituting equations (14) and (15) into the homogeneous parts of equations (10) and (11) results in the equations

$$
\frac{\partial G}{\partial r}+\frac{1}{r} \frac{\partial H}{\partial \eta}=0, \quad \frac{1}{r} \frac{\partial G}{\partial \eta}-\frac{\partial H}{\partial r}=0
$$




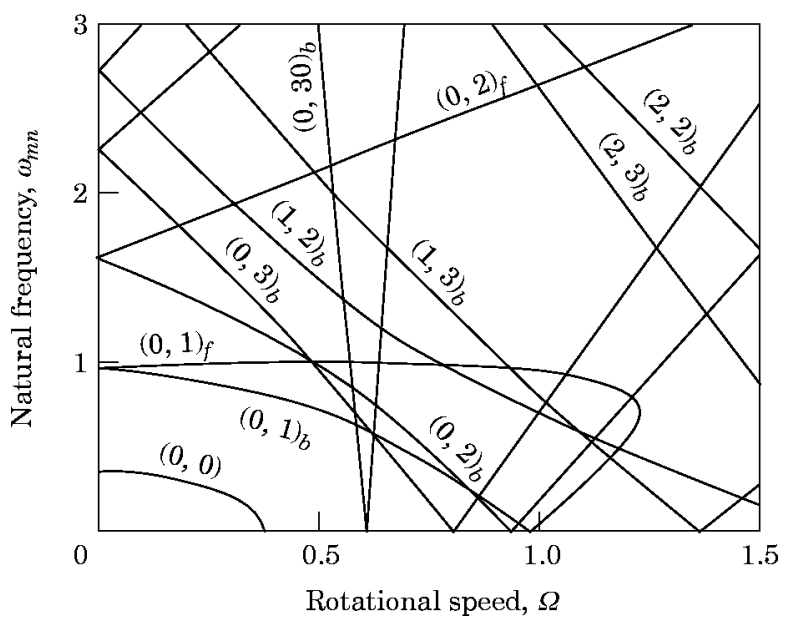

Figure 2. The natural frequencies of in-plane vibration of an annular disk with clamping ratio $0 \cdot 2$.

where

$$
\begin{aligned}
& G=\lambda_{1}^{2} \nabla^{2} \phi-\Omega^{2} \partial^{2} \phi / \partial \eta^{2}+\Omega^{2} \phi-2 \Omega^{2} \partial \psi / \partial \eta-\partial^{2} \phi / \partial t^{2}-2 \Omega \partial^{2} \phi / \partial t \partial \eta-2 \Omega \partial \psi / \partial t, \\
& H=\lambda_{2}^{2} \nabla^{2} \psi-\Omega^{2} \partial^{2} \psi / \partial \eta^{2}+\Omega^{2} \psi+2 \Omega^{2} \partial \phi / \partial \eta-\partial^{2} \psi / \partial t^{2}-2 \Omega \partial^{2} \psi / \partial t \partial \eta+2 \Omega \partial \phi / \partial t,
\end{aligned}
$$

and, $\lambda_{1}$ and $\lambda_{2}$ are two constants depending on the Poisson ratio,

$$
\lambda_{1}^{2}=1 /\left(1-v^{2}\right), \quad \lambda_{2}^{2}=1 / 2(1+v) .
$$

The general solutions for $G$ and $H$ in equations (16) and (17) can be expressed in the form

$$
G=\left(A_{n} r^{n}+B_{n} r^{-n}\right) \cos n \eta, \quad H=\left(-A_{n} r^{n}+B_{n} r^{-n}\right) \sin n \eta, \quad n=0,1,2, \ldots,
$$

where $A_{n}$ and $B_{n}$ are undetermined constants. Equations (18) and (19) are two inhomogeneous equations for $\phi$ and $\psi$. The complete solutions for $\phi$ and $\psi$ include time-independent particular solutions $\phi_{p}, \psi_{p}$, and time-dependent homogeneous solutions $\phi_{h}$ and $\psi_{h}$. Both the homogeneous and the particular solutions should satisfy the homogeneous boundary conditions (12) and (13). While $\phi_{p}$ and $\psi_{p}$ are important in determining the steady state response [5], they have nothing to do with the natural frequencies of the system. In order for the homogeneous solutions to satisfy the homogeneous boundary conditions, $\phi_{h}$ and $\psi_{h}$ may be expressed in the forms

$$
\phi_{h}(r, \eta ; t)=\Phi_{m n}(r) \mathrm{e}^{\mathrm{i} m \eta} \mathrm{e}^{-i \omega_{m n} t}, \quad \psi_{h}(r, \eta ; t)=-\mathrm{i} \Psi_{m n}(r) \mathrm{e}^{\mathrm{i} m \eta} \mathrm{e}^{-\mathrm{i} \omega_{m n} t},
$$

where

$$
\begin{aligned}
& \Phi_{m n}(r)=c_{n} \mathbf{J}_{n}\left(\beta_{n 1} r\right)+d_{n} s_{n 1} \mathbf{J}_{n}\left(\beta_{n 2} r\right)+e_{n} \mathrm{Y}_{n}\left(\beta_{n 1} r\right)+f_{n} s_{n 1} Y_{n}\left(\beta_{n 2} r\right), \\
& \Psi_{m n}(r)=c_{n} s_{n 2} \mathrm{~J}_{n}\left(\beta_{n 1} r\right)+d_{n} \mathbf{J}_{n}\left(\beta_{n 2} r\right)+e_{n} s_{n 2} Y_{n}\left(\beta_{n 1} r\right)+f_{n} Y_{n}\left(\beta_{n 2} r\right) .
\end{aligned}
$$


Here $J$ and $\mathrm{Y}$ are Bessel functions of the first and the second kinds. $\omega_{m n}$ is a natural frequency of the spinning disk. $s_{n 1}$ and $s_{n 2}$ are defined as

$$
s_{n 1}=\frac{-2 \Omega\left(\omega_{m n}-n \Omega\right)}{(\omega-n \Omega)^{2}+\Omega^{2}-\lambda_{1}^{2} \beta_{n 2}^{2}} \quad \text { and } \quad s_{n 2}=\frac{-2 \Omega\left(\omega_{m n}-n \Omega\right)}{(\omega-n \Omega)^{2}+\Omega^{2}-\lambda_{2}^{2} \beta_{n 1}^{2}},
$$

where, $\beta_{n 1}$ and $\beta_{n 2}$ are positive real roots of the quartic equation

$$
\lambda_{1}^{2} \lambda_{2}^{2} \beta_{n}^{4}-\left(\lambda_{1}^{2}+\lambda_{2}^{2}\right) \beta_{n}^{2}\left[\left(\omega_{m n}-n \Omega\right)^{2}+\Omega^{2}\right]+\left[\left(\omega_{m n}-n \Omega\right)^{2}+\Omega^{2}\right]^{2}=0 .
$$

After applying the homogeneous boundary conditions $(11,12)$, the resulting frequency equation can be obtained as

$$
\left|\begin{array}{llll}
g_{11} & g_{12} & g_{13} & g_{14} \\
g_{21} & g_{22} & g_{23} & g_{24} \\
g_{31} & g_{32} & g_{33} & g_{34} \\
g_{41} & g_{42} & g_{43} & g_{44}
\end{array}\right|=0,
$$

where the $g_{i j}$ are defined in the Appendix. For each $n$, there is an infinite number of discrete $\omega_{m n}$ which satisfy equation (29).

\section{NATURAL FREQUENCIES AND CRITICAL SPEEDS}

In Figures 2-4 are shown the dimensionless natural frequencies $\omega_{m n}$ as functions of the dimensionless rotational speed $\Omega$ for clamping ratios $\zeta=0 \cdot 2,0 \cdot 5$ and $0 \cdot 7$, respectively. The model label $(m, n)_{f}$ denotes the forward traveling modes with $m$ modal circles and $n$ nodal diameters. Similarly, the subscript $b$ denotes a backward traveling wave. The critical speed $\Omega_{c n}$ is defined as the rotational speed at which the natural frequency of the mode $(0, n)_{b}$ vanishes. The numerical results of our calculations are partially checked by two previous papers, one of which is the paper by Burdess et al. [3] on the in-plane vibration of a full disk with two nodal diameters. Our results approach those in reference [3] as the clamping ratio approaches zero. The second check is to compare our results with those presented by Srinivasan and Ramamurti [6] on a non-rotating annular disk with clamping ratio ranging from $0 \cdot 2$ to $0 \cdot 8$. We proceed with our analysis after verifying our results by successful comparisons with these previous works.

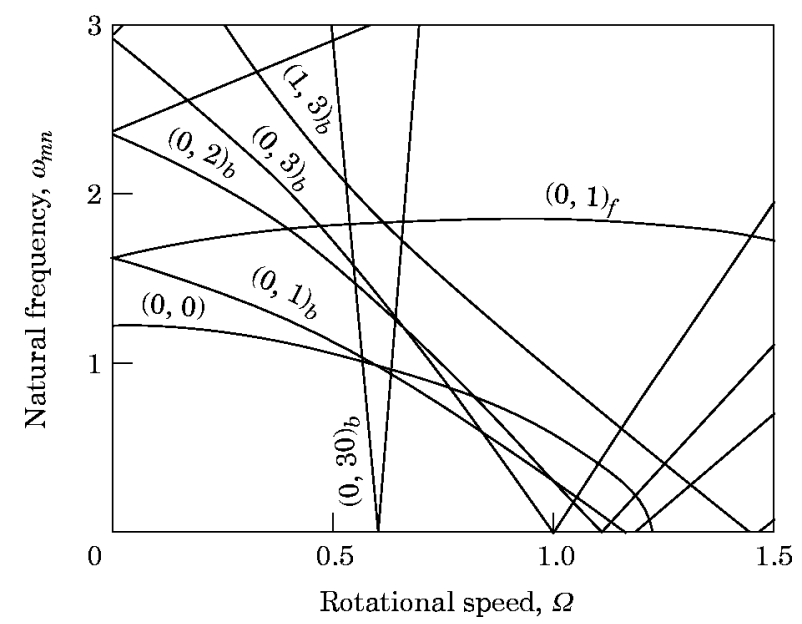

Figure 3. The natural frequencies of in-plane vibration of an annular disk with clamping ratio $0 \cdot 5$. 


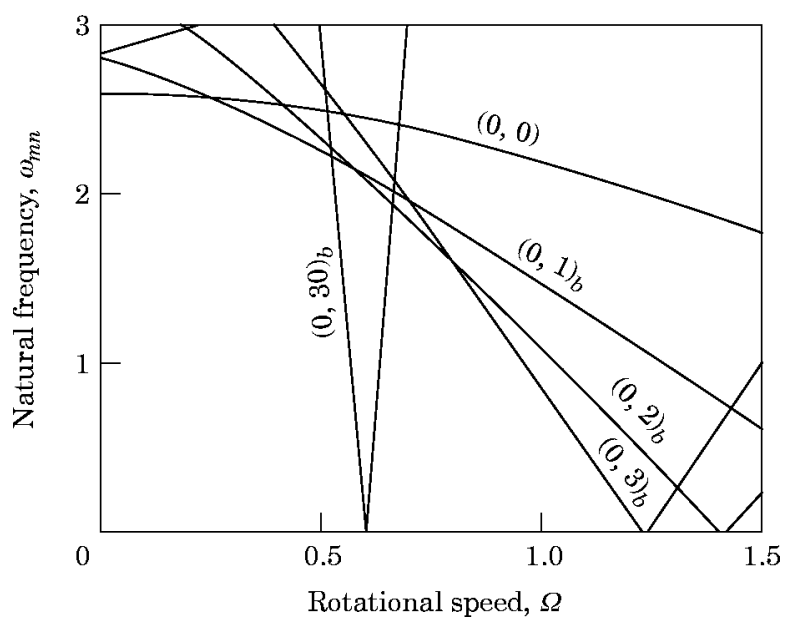

Figure 4. The natural frequencies of in-plane vibration of an annular disk with clamping ratio $0 \cdot 7$.

In Figures 2-4 we show only the natural frequency loci of the modes with $n=0,1,2,3$ and 30. The natural frequency loci of the modes with $n=4,5, \ldots, 29$ are omitted in these figures for brevity, with the understanding that the slopes of these curves increase as $n$ increases, and the corresponding critical speeds move leftward toward the critical speed corresponding to the mode $(0,30)_{b}$. For the modes with $n>30$, which are also not shown in these figures, the corresponding critical speeds continue to move leftward slightly and approach an asymptotic value.

In Figure 5 is shown the relation between the critical speed $\Omega_{c n}$ and the number of nodal diameters $n$ for a Poisson ratio of 0.3 and for clamping ratios $0.1,0.3,0.5,0.7$ and 0.9 . This figure is obtained by specifying $\omega=0$ in equations (27) and (28), and solving for $\Omega$ from equation (29) for various $n$. It is clear from this figure that $\Omega_{c n}$ indeed approaches an asymptotic values $(0.572$ for $n=300)$ as $n$ increases. Furthermore, it is interesting to observe that the asymptotic critical speed is independent of the clamping ratio. The relation between the critical speed of the mode $(0,200)$ and the Poisson ratio $v$ is shown in Figure 6. No appreciable difference is observed in Figure 6 if $n=300$ is used in the calculation instead of 200.

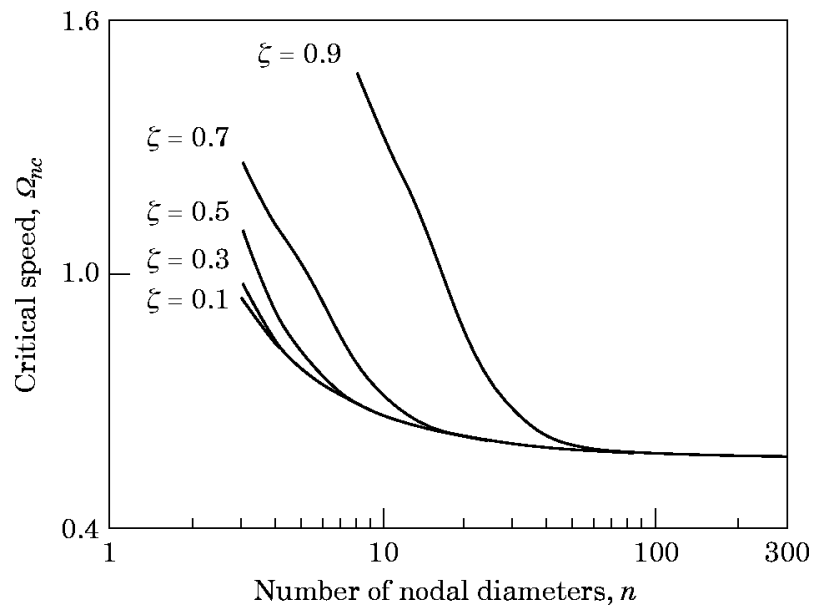

Figure 5. The relation between the critical speed and the number of nodal diameters. 


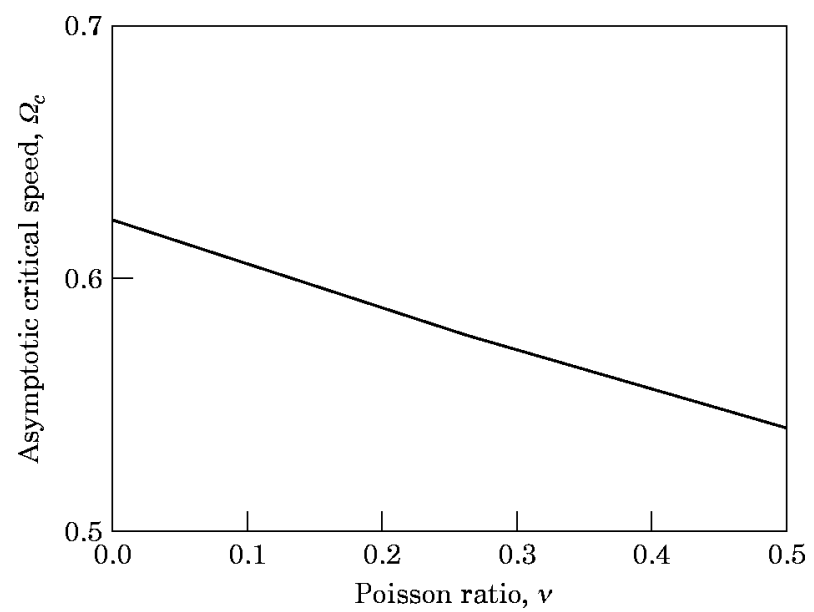

Figure 6. The relation between the critical speed of the mode $(0,200)$ and the Poisson ratio $v$.

It is also interesting to compare the critical speeds of the in-plane vibration with those of the out-of-plane vibration of a spinning disk (see Figure 6 of reference [8]). In the case of a large clamping ratio, the critical speeds in both cases tend to be dominated by the modes with a large number of nodal diameters.

The phenomenon that the critical speeds of the modes with a large number of nodal diameters tend to approach an asymptotic value may be better understood if one looks at the equation of motion (10) carefully. For the modes with very large $n$ the terms with a second order derivative with respect to $\eta$ tend to dominate because the mode shapes are known to be of the forms (23) and (24). After retaining only these terms with $\partial^{2} / \partial \eta^{2}$ one obtains the equation

$$
\{1 / 2(1+v)\}(1 / r) \partial^{2} u_{r} / \partial \eta^{2}=\Omega^{2} \partial^{2} u_{r} / \partial \eta^{2} .
$$

Therefore, one may estimate the limiting critical speed for a very large number of nodal diameters roughly, bearing in mind that this limiting speed is independent of the clamping ratio, as $\Omega_{c} \approx 1 / \sqrt{2(1+v)}$.

One of the dynamic systems exhibiting a similar phenomenon is the lateral vibration of a rotating circular string [7], with an equation of motion in the form

$$
\partial^{2} u / \partial t^{2}+2 \Omega \partial^{2} u / \partial t \partial \eta-\left(s^{2}-\Omega^{2}\right) \partial^{2} u / \partial \eta^{2}=0,
$$

where $u$ is the lateral displacement of the rotating string with respect to the inertial frame, and $s$ is proportional to the string tension. In this case, the critical speeds of all modes are exactly equal to $s$. Sometimes this phenomenon is attributed to the non-dispersive nature of the string, in contrast to those dispersive systems such as beams or plates. Of course, the in-plane vibration of a spinning disk is dispersive in nature. However, it may be said that the dispersive nature of the vibration modes with a large number of nodal diameters tends to be diminishing.

The speed limit to which the disk is allowed to rotate safely depends on the clamping ratio, however. For the disk with clamping ratio $\zeta=0 \cdot 2$, the first critical speed corresponds to the mode $(0,0)$ and is equal to $0 \cdot 369$, as shown in Figure 2 . Beyond this critical speed the natural frequency $\omega_{00}$ becomes purely imaginary and divergence instability is induced. A closer look at the corresponding eigenfunctions reveals that the mode $(0,0)$ is a torsional mode, while the mode $(1,0)$ is a radial mode. As the clamping ratio increases, $\zeta=0 \cdot 7$ for instance, the asymptotic critical speed becomes the limit speed of the spinning disk. 


\section{CONCLUSIONS}

The effects of the clamping ratio on the critical speeds and the natural frequencies of in-plane vibration of a spinning annular disk have been investigated. For a disk with a small clamping ratio, the limiting speed at which the disk is allowed to rotate safely is determined by the critical speed of the axisymmetrical mode. For the cases with a large clamping ratio, on the other hand, the limiting speed is determined by the critical speed of modes with a large number of nodal diameters. Numerical simulation shows that the critical speeds of the modes with nodal diameters approach an asymptotic value as the number of nodal diameters increases. This asymptotic critical speed appears to be independent of the clamping ratio, while it is dependent on the Poisson ratio of the disk.

\section{REFERENCES}

1. P. G. Bhuta and J. P. Jones 1963 Journal of the Acoustical Society of America 35(7), 982-989. Symmetric planar vibrations of a rotating disc.

2. R. DoBy 1969 Journal of the Franklin Institute 288(3), 203-212. On the elastic stability of Coriolis-coupled oscillations of a rotating disc.

3. J. S. Burdess, T. Wren and J. N. FawCEtT 1987 Proceedings of the Institution of Mechanical Engineers 201, 37-44. Plane stress vibrations in rotating discs.

4. A. C. Eringen and W. S. Suhubi 1975 Elastodynamics (Vol. II). New York: Academic Press.

5. J.-L. JHU 1995 Master's Thesis, Department of Mechanical Engineering, National Taiwan University. In-plane stress and displacement distributions in a spinning annular disk under stationary edge loads.

6. V. SRinivasan and V. Ramamurti 1980 Journal of Sound and Vibration 72, 251-262. Dynamic response of an annular disk to a moving concentrated, in-plane edge load.

7. G. S. SCHAJER 1984 Journal of Sound and Vibration 92, 11-19. The vibration of a rotating circular string subject to a fixed elastic restraint.

8. G. Adams 1987 International Journal of Mechanical Sciences 29(8), 525-531. Critical speeds for a flexible spinning disk.

$$
\begin{gathered}
\text { APPENDIX } \\
g_{11}=\kappa_{n 8} \zeta^{-1} \mathbf{J}_{n}\left(\beta_{n 1} \zeta\right)-\beta_{n} \mathbf{J}_{n+1}\left(\beta_{n 1} \zeta\right), \quad g_{12}=\kappa_{n 7} \zeta^{-1} \mathbf{J}_{n}\left(\beta_{n 2} \zeta\right)-s_{n 1} \beta_{n 2} \mathbf{J}_{n+1}\left(\beta_{n 2} \zeta\right), \\
g_{13}=\kappa_{n 8} \zeta^{-1} Y_{n}\left(\beta_{n 1} \zeta\right)-\beta_{n 1} Y_{n+1}\left(\beta_{n 1} \zeta\right), \quad g_{14}=\kappa_{n 7} \zeta^{-1} \mathbf{Y}_{n}\left(\beta_{n 2} \zeta\right)-s_{n 1} \beta_{n 2} \mathbf{Y}_{n+1}\left(\beta_{n 2} \zeta\right), \\
g_{21}=-\kappa_{n 8} \zeta^{-1} \mathbf{J}_{n}\left(\beta_{n 1} \zeta\right)+s_{n 2} \beta_{n 1} \mathbf{J}_{n+1}\left(\beta_{n 1} \zeta\right), \quad g_{22}=-\kappa_{n 7} \zeta^{-1} \mathbf{J}_{n}\left(\beta_{n 2} \zeta\right)+\beta_{n 2} \mathbf{J}_{n+1}\left(\beta_{n 2} \zeta\right), \\
g_{23}=-\kappa_{n 8} \zeta^{-1} Y_{n}\left(\beta_{n 1} \zeta\right)+s_{n 2} \beta_{n 1} Y_{n+1}\left(\beta_{n 1} \zeta\right), \quad g_{24}=-\kappa_{n 7} \zeta^{-1} Y_{n}\left(\beta_{n 2} \zeta\right)+\beta_{n 2} Y_{n+1}\left(\beta_{n 2} \zeta\right), \\
g_{31}=\left(\kappa_{n 1}-\beta_{n 1}^{2}\right) \mathbf{J}_{n}\left(\beta_{n 1}\right)+\kappa_{n 2} \mathbf{J}_{n+1}\left(\beta_{n 1}\right), \\
g_{32}=\left(\kappa_{n 3}-s_{n 1} \beta_{n 2}^{2}\right) \mathbf{J}_{n}\left(\beta_{n 2}\right)+\kappa_{n 4} \mathbf{J}_{n+1}\left(\beta_{n 2}\right), \\
g_{33}=\left(\kappa_{n 1}-\beta_{n 1}^{2}\right) Y_{n}\left(\beta_{n 1}\right)+\kappa_{n 2} Y_{n+1}\left(\beta_{n 1}\right), \\
g_{34}=\left(\kappa_{n 1}-\frac{1}{2} s_{n 2} \mu_{0} \beta_{n 1}^{2}\right) \mathbf{J}_{n}\left(\beta_{n 1}\right)+\kappa_{n 5} \mathbf{J}_{n+1}\left(\beta_{n 1}\right), \\
g_{42}=\left(\kappa_{n 3}-\frac{1}{2} \mu_{0} \beta_{n 2}^{2}\right) \mathbf{J}_{n}\left(\beta_{n 2}\right)+\kappa_{n 6} \mathbf{J}_{n+1}\left(\beta_{n 2}\right), \\
g_{43}=\left(\kappa_{n 1}-\frac{1}{2} s_{n 2} \mu_{0} \beta_{n 1}^{2}\right) Y_{n}\left(\beta_{n 1}\right)+\kappa_{n 5} Y_{n+1}\left(\beta_{n 1}\right), \\
g_{44}=\left(\kappa_{n 3}-\frac{1}{2} \mu_{0} \beta_{n 2}^{2}\right) Y_{n}\left(\beta_{n 2}\right)+\kappa_{n 6} Y_{n+1}\left(\beta_{n 2}\right) .
\end{gathered}
$$


the constants $\kappa_{n i}$ and $\mu_{0}$ are defined as follows:

$$
\begin{gathered}
\kappa_{n 1}=\mu_{0}\left(1+s_{n 2}\right)\left(n^{2}-n\right), \quad \kappa_{n 2}=\mu_{0}\left(1-n s_{n 2}\right) \beta_{n 1}, \quad \kappa_{n 3}=\mu_{0}\left(1+s_{n 1}\right)\left(n^{2}-n\right), \\
\kappa_{n 4}=-\mu_{0}\left(n-s_{n 1}\right) \beta_{n 2}, \\
\kappa_{n 5}=-\mu_{0}\left(n-s_{n 2}\right) \beta_{n 1}, \quad \kappa_{n 6}=\mu_{0}\left(1-n s_{n 1}\right) \beta_{n 2}, \quad \kappa_{n 7}=n\left(1+s_{n 1}\right), \\
\kappa_{n 8}=n\left(1+s_{n 2}\right), \quad \mu_{0}=1-v .
\end{gathered}
$$

